\title{
Clinical experience with integrase inhibitors in HIV-2-infected individuals in Spain
}

\author{
S. Requena ${ }^{1}$, A. B. Lozano ${ }^{2}$, E. Caballero ${ }^{3}$, F. García ${ }^{4}$, M. C. Nieto ${ }^{5}$, R. Téllez ${ }^{6}$, J. M. Fernández ${ }^{2}$, \\ M. Trigo ${ }^{7}$, I. Rodríguez-Avial ${ }^{8}$, L. Martín-Carbonero ${ }^{9}$, P. Miralles ${ }^{10}$, V. Soriano ${ }^{9,11}$ and \\ C. de Mendoza ${ }^{1,12 *}$ on behalf of the HIV-2 Spanish Study Group†
}

\begin{abstract}
${ }^{1}$ Puerta de Hierro University Hospital and Research Institute, Madrid, Spain; ${ }^{2}$ Hospital de Poniente, Almeria, Spain; ${ }^{3}$ Hospital Vall d'Hebró, Barcelona, Spain; ${ }^{4}$ Hospital Universitario San Cecilio, Instituto de Investigación Ibs, Granada, Spain; ${ }^{5}$ Hospital de Basurto, Bilbao, Spain; ${ }^{6}$ Fundación Jiménez-Díaz, Madrid, Spain; ${ }^{7}$ Complejo Hospitalario, Pontevedra, Spain; ${ }^{8}$ Hospital Clínico San Carlos, Madrid, Spain; ${ }^{9}$ Hospital Universitario La Paz, Madrid, Spain; ${ }^{10}$ Hospital Universitario Gregorio Marañón, Madrid, Spain; ${ }^{11}$ UNIR Health Sciences School, Madrid, Spain; ${ }^{12}$ Universidad San Pablo CEU, Madrid, Spain
*Corresponding author. Internal Medicine Department, Puerta de Hierro University Hospital \& Research Institute, Majadahonda, Madrid, Spain. E-mail: cmendoza.cdm@gmail.com
†Members are listed in the Acknowledgements section.

Received 22 October 2018; returned 28 November 2018; revised 26 December 2018; accepted 31 December 2018

Background: HIV-2 is a neglected virus despite estimates of 1-2 million people being infected worldwide. The virus is naturally resistant to some antiretrovirals used to treat HIV-1 and therapeutic options are limited for patients with HIV-2.

\begin{abstract}
Methods: In this retrospective observational study, we analysed all HIV-2-infected individuals treated with integrase strand transfer inhibitors (INSTIs) recorded in the Spanish HIV-2 cohort. Demographics, treatment modalities, laboratory values, quantitative HIV-2 RNA and CD4 counts as well as drug resistance were analysed.

Results: From a total of 354 HIV-2-infected patients recruited by the Spanish HIV-2 cohort as of December 2017, INSTIs had been given to 44, in 18 as first-line therapy and in 26 after failing other antiretroviral regimens. After a median follow-up of 13 months of INSTI-based therapy, undetectable viraemia for HIV-2 was achieved in $89 \%$ of treatment-naive and in $65.4 \%$ of treatment-experienced patients. In parallel, CD4 gains were 82 and 126 cells $/ \mathrm{mm}^{3}$, respectively. Treatment failure occurred in 15 patients, 2 being treatment-naive and 13 treatment-experienced. INSTI resistance changes were recognized in 12 patients: N155H (5), Q148H/R (3), Y143C/G (3) and R263K (1).
\end{abstract}

Conclusions: Combinations based on INSTIs are effective and safe treatment options for HIV-2-infected individuals. However, resistance mutations to INSTIs are selected frequently in failing patients, reducing the already limited treatment options.

\section{Introduction}

HIV-2 was first described in 1986 in two patients from West Africa presenting with AIDS. ${ }^{1}$ Current estimates indicate $1-2$ million people infected with HIV-2 worldwide, including dual HIV-1 plus HIV-2 coinfections. ${ }^{2}$ In contrast to the global spread of HIV-1, HIV-2 has remained largely confined to some countries in West Africa where it is endemic. ${ }^{3}$ In the EU, HIV-2 has been introduced by the large immigration flow from Sub-Saharan countries.

A national registry of HIV-2 cases has existed in Spain since 1989, shortly after the first individuals with HIV-2 infection were identified. They were three males of West African origin who had recently arrived and were living in north Barcelona. ${ }^{4}$ Since then, a total of 354 cases of HIV-2 infection have been reported to the Spanish HIV-2 registry, of which $63 \%$ are in males. Whereas $72 \%$ are Sub-Saharan Africans, $16 \%$ are native Spaniards. Although most cases are found around the largest urban areas (Barcelona and Madrid), two further foci of HIV-2 have been found in Galicia, in the northwest and in Almeria, in the southeast coast of Spain, most likely associated with sailors working in West Africa and the recent arrival of illegal boats, respectively. ${ }^{5}$

ART for HIV-2 lags far behind HIV-1 therapeutics, owing to the fact that the drugs have been designed using HIV-1 enzyme structures. Protein variability in HIV-2 explains the poor lack of binding and inhibitory effect of some of these agents. ${ }^{6}$ In this regard, 
HIV-2 is non-susceptible to NNRTIs and fusion inhibitors. ${ }^{7,8}$ Moreover, several protease inhibitors show weak or no inhibitory activity against HIV-2. 7,9,10

All integrase strand transfer inhibitors (INSTIs) approved for the treatment of HIV-1 are similarly active against HIV-2. ${ }^{11,12}$ Moreover, drug resistance mutations in patients with HIV-2 failing on INSTIs tend to be selected at the same positions in both viruses. ${ }^{7,13-16}$ However, the information available about the use of INSTIs in HIV-2-infected individuals is scarce and limited to small numbers of patients. In this nationwide observational study, we analysed the clinical and virological outcome of all HIV-2-infected individuals treated with INSTIs in Spain.

\section{Patients and methods}

The Spanish HIV-2 national register is a database that has collected information from individuals diagnosed with HIV-2 infection across the country since the registry began in $1989 .{ }^{5}$ In addition, a centralized laboratory repository of stored clinical samples, including peripheral blood mononuclear cells and plasma, exists for all HIV-2-infected individuals on regular follow-up, providing virological information, including subtyping and drug resistance mutations. ${ }^{17}$

All HIV-2-infected individuals who had initiated ART with INSTI-based regimens were identified. We retrospectively analysed demographics, treatment modalities, laboratory values, quantitative plasma HIV-2 RNA and CD4 counts as well as drug resistance mutations in patients failing ART. Treatment success was defined as achievement of undetectable plasma viraemia (HIV-2 RNA $<50$ copies $/ \mathrm{mL}$ ) at any time. In contrast, treatment failure was defined as lack of achievement of undetectable viral load 12 weeks after beginning therapy, or viral rebound after reaching undetectability.

Plasma HIV-2 RNA was measured using a non-commercial validated, real-time PCR assay. The region amplified was the long terminal repeat with primers and probes described elsewhere. ${ }^{18}$ The limit of detection was HIV-2 RNA 50 copies/mL. Both HIV-2 groups A and B are reliably detected with this assay.

Amplification of sequences within the polymerase region (protease, RT and integrase) was attempted in plasma specimens. For HIV-2 RNA extraction, plasma was processed as indicated on the RNA extraction kit used (Abbott sample preparation system RNA, Spain). Primers and conditions have been previously described. ${ }^{13,19}$ The product was purified (QIAquick PCR Purification Kit; QIAGEN, Germany) and finally sequenced using PCR [BigDye Terminator v1.1, v3.1 5x Sequencing Buffer (Applied Biosystems, UK) and BigDye Terminator v1.1 Cycle (Applied Biosystems, USA)]. Bulk Sanger sequencing was carried out using the 3100-Avant Genetic Analyzer (Applied Biosystems, USA). Sequences were analysed using SeqScape V2.5 using HIV-2 ROD as the reference strain.

Drug resistance-associated changes along with compensatory drug resistance mutations were considered using the information available for HIV-1 and HIV-2 at the Stanford HIV-1 drug resistance database, ${ }^{20}$ the 2011 International AIDS Society-USA panel mutation list ${ }^{21}$ and the HIV-2 EU-supporting resistance rules. ${ }^{22}$

\section{Statistical analysis}

All figures are recorded numerically as absolute values and percentages. Comparisons between drug-naive and antiretroviral-experienced patients were performed using $\chi^{2}$ or Fisher's exact tests. All statistical analyses were performed using SPSS software version 20.0 (IBM Corp., Armonk, NY, USA).

\section{Results}

From 354 HIV-2-infected individuals recorded at the HIV-2 Spanish cohort up to December 2017, a total of 44 had been
Table 1. Main demographics of the HIV-2 study population treated with integrase inhibitors

\begin{tabular}{|c|c|c|c|}
\hline Characteristic & Total & $\begin{array}{l}\text { Antiretroviral- } \\
\text { naive }\end{array}$ & $\begin{array}{l}\text { Treatment- } \\
\text { experienced }\end{array}$ \\
\hline No. & 44 & 18 & 26 \\
\hline $\begin{array}{l}\text { Median age at diagnosis, } \\
\text { years }\end{array}$ & $43(37-50)$ & $44.5(38-53)$ & $43(34-49)$ \\
\hline \multicolumn{4}{|l|}{ Gender, n (\%) } \\
\hline male & $32(72.7)$ & 10 & 22 \\
\hline female & $12(27.3)$ & 8 & 4 \\
\hline \multicolumn{4}{|l|}{ Risk group, $n(\%)$} \\
\hline heterosexual & $31(70.5)$ & 13 & 18 \\
\hline homosexual & $1(2.3)$ & - & 1 \\
\hline vertical & $1(2.3)$ & - & 1 \\
\hline unknown & $11(25)$ & 5 & 6 \\
\hline \multicolumn{4}{|l|}{ Origin, $n(\%)$} \\
\hline Africa & $36(81.8)$ & 14 & 22 \\
\hline Spain & $5(11.4)$ & 2 & 3 \\
\hline Portugal & $2(4.5)$ & 1 & 1 \\
\hline Brazil & $1(2.3)$ & 1 & - \\
\hline \multicolumn{4}{|l|}{ Year of diagnosis, $n$ (\%) } \\
\hline$<2014$ & $32(72.7)$ & 8 & 24 \\
\hline$\geq 2014$ & $12(27.3)$ & 10 & 2 \\
\hline HIV-1 coinfection, $n$ (\%) & $5(11.4)$ & 3 & 2 \\
\hline
\end{tabular}

treated with an INSTI, 18 being antiretroviral-naive and 26 having had virological failure under another regimen. The main characteristics of the study population are recorded in Table 1. Overall, $72.7 \%$ of these 44 individuals were male, with a median age of 43 years. More than $80 \%$ came from Sub-Saharan Africa and $70 \%$ had acquired HIV-2 infection through heterosexual contacts. Coinfection with HIV-1 had been demonstrated in five individuals. There were no significant differences in demographics when comparing individuals who started INSTI as first-line therapy and those who were treated with INSTI as a rescue intervention.

From the 18 drug-naive HIV-2 individuals who started treatment with INSTI, nine received raltegravir, six elvitegravir and three dolutegravir. Nucleoside analogues included as backbone were mostly tenofovir with either emtricitabine or lamivudine. One patient also included darunavir/ritonavir in his initial treatment regimen (Table 2). Median baseline CD4 counts and HIV-2 viral load were 264 cells $/ \mathrm{mm}^{3}$ and $3.6 \mathrm{log}$ copies $/ \mathrm{mL}$, respectively. However, nearly half of individuals had undetectable plasma viraemia before beginning treatment. After a median follow-up of 12 months, 16 $(88.9 \%)$ individuals achieved and/or maintained undetectable HIV-2 viraemia. The median CD4 gain was 82 cells $/ \mathrm{mm}^{3}$.

A total of 26 individuals received INSTI as part of a rescue intervention. They received raltegravir (19), dolutegravir (6) and elvitegravir (1). Moreover, all received two nucleoside analogues and 13 individuals included a boosted PI in the new regimen, with this being darunavir/ritonavir in 10 of them (Table 2). The median CD4 count before beginning INSTI was 194 cells $/ \mathrm{mm}^{3}$ and the median plasma HIV-2 RNA was 3.7 log copies/mL in viraemic subjects. Of note, roughly one-third of patients had undetectable HIV-2 RNA at the time of shifting to INSTI, based on the clinical decision of the 
Table 2. Main clinical characteristics of patients with HIV-2 treated with integrase inhibitors

\begin{tabular}{|c|c|c|}
\hline Characteristic & Antiretroviral-naive & Treatment-experienced \\
\hline No. & 18 & 26 \\
\hline Baseline CD4 counts, cells $/ \mathrm{mm}^{3}$, median (IQR) & $264(134-604)$ & $194(52-421)$ \\
\hline \multicolumn{3}{|l|}{ Plasma HIV-2 RNA } \\
\hline baseline plasma HIV-2 RNA undetectable, $n$ (\%) & $8(44.4 \%)$ & $10(38.5 \%)$ \\
\hline VL in viraemic patients, log copies/mL, median (IQR) & $3.6(2.6-4.2)$ & $3.7(2.7-4.4)$ \\
\hline \multicolumn{3}{|l|}{ HIV-2 subtype, $n(\%)$} \\
\hline A & 7 & 17 \\
\hline$B$ & 1 & 4 \\
\hline unknown & 10 & 5 \\
\hline \multicolumn{3}{|l|}{ Integrase inhibitors, n (\%) } \\
\hline raltegravir & $9(50 \%)$ & $19(73.1 \%)$ \\
\hline elvitegravir & $6(33.3 \%)$ & $1(3.8 \%)$ \\
\hline dolutegravir & $3(16.7 \%)$ & $6(23.1 \%)$ \\
\hline \multicolumn{3}{|l|}{ Protease inhibitors, $n(\%)$} \\
\hline darunavir/ritonavir ${ }^{a}$ & $1(5.6 \%)$ & $10(38.5 \%)$ \\
\hline others & 0 & $3^{\mathrm{b}}(11.5 \%)$ \\
\hline \multicolumn{3}{|l|}{ Nucleoside analogues, $n$ (\%) } \\
\hline tenofovir alafenamide + emtricitabine & $4(22.2 \%)$ & $1(3.8 \%)$ \\
\hline tenofovir disoproxil fumarate + lamivudine or emtricitabine & $11(61.1 \%)$ & $17(65.4 \%)$ \\
\hline abacavir + lamivudine & $3(16.7 \%)$ & $3(11.5 \%)$ \\
\hline none & 0 & 5 \\
\hline Patients with undetectable VL at any time during follow-up, $n$ (\%) & $16(88.9 \%)$ & $17^{\mathrm{C}}(65.4 \%)$ \\
\hline Time with undetectable VL, months, median (IQR) & $12(6-29)$ & $13(0-27.5)$ \\
\hline Gain in CD4 counts, cells $/ \mathrm{mm}^{3}$, median (IQR) & $82(13-272)$ & $126(48-200)$ \\
\hline Treatment failure on integrase inhibitor, $n(\%)$ & $2(11.1 \%)$ & $13(50 \%)$ \\
\hline
\end{tabular}

VL, viral load.

${ }^{a} 600 \mathrm{mg} / 100 \mathrm{mg}$ twice daily.

bone lopinavir/ritonavir; one tipranavir/ritonavir; one saquinavir/ritonavir.

${ }^{\mathrm{C}}$ Four individuals achieved undetectable viraemia after beginning integrase inhibitor therapy but VL rebounded during follow-up.

doctor in charge, considering poor CD4 response as the reason for failing the prior regimen. A total of $17(65.4 \%)$ individuals achieved undetectable viraemia for a median of 13 months. However, four baseline viraemic patients experienced relapse during further follow-up. Overall, the median CD4 count gain in this population was 126 cells $/ \mathrm{mm}^{3}$.

Virological failure under INSTI-based therapy was recognized in 15 HIV-2-infected individuals, 2 being (11.1\%) drug-naive and 13 (50\%) treatment-experienced. Of them, only four had achieved undetectable viral load at some point during therapy, experiencing relapse shortly after. The remaining 11 patients did not reach undetectable viraemia at any timepoint. The mean time between initiation of INSTI-based therapy and genotypic analysis at failure was 42 weeks (range 16-88).

A total of 12 individuals developed INSTI-associated resistance mutations N155H (5), Q148H/R (3), Y143C/G (3) and R263K (1). All but one developed other compensatory changes (Table 3). For the RT viral region, seven individuals developed M184V, six K65R and one Q151M. Combinations of these mutations were seen in three cases, one being $K 65 R+Q 151 M$ and two $\mathrm{K} 65 \mathrm{R}+\mathrm{M} 184 \mathrm{~V}$. In addition, substitutions were recognized at the protease in 10 patients, as follows: I50V (3); I54L/M (6); I82F/L (5); I84V (3); and L90M (3). In eight of them, these changes reflected mutations persisting after failing previously on PI-based therapies.

\section{Discussion}

The treatment of HIV-2-infected individuals generally follows the rules of HIV-1 with a few special considerations. ${ }^{23-25}$ However, to date no information drawn from randomized controlled trials guides the timing for ART initiation in patients with HIV-2, and there are some conflicting opinions. ${ }^{11}$ Thus, the optimal treatment strategy for HIV-2 infection remains unclear. Current guidelines for HIV-2-infected individuals are based on retrospective cohort studies, small case series, individual case reports, in vitro data and extrapolation from studies conducted in patients with HIV-1. ${ }^{6,25}$

All INSTIs approved to date for HIV-1 exhibit potent activity against HIV-2. ${ }^{26-28}$ Two recent clinical trials conducted in France and Senegal, respectively, evaluated the efficacy and safety of raltegravir and elvitegravir as first-line ART in HIV-2-infected individuals. The first study, conducted by Matheron et al., ${ }^{29}$ assessed the combination of raltegravir/emtricitabine/tenofovir in $30 \mathrm{HIV}-2$ infected patients. In terms of virological response, 27 of 28 participants who completed the 48 week follow-up achieved $<40$ copies $/ \mathrm{mL}$ with a median CD 4 gain of +87 cells $/ \mathrm{mm}^{3}$. However, only $40 \%$ of 
Table 3. Main characteristics of patients with HIV-2 that failed virologically under integrase inhibitors

\begin{tabular}{|c|c|c|c|c|c|c|c|c|}
\hline \multirow{2}{*}{$\begin{array}{l}\text { Patient } \\
\text { no. }{ }^{a}\end{array}$} & \multirow{2}{*}{$\begin{array}{c}\text { Gender } \\
\text { (age, years) }\end{array}$} & \multirow{2}{*}{$\begin{array}{c}\text { Country of } \\
\text { origin }\end{array}$} & \multirow{2}{*}{$\begin{array}{c}\text { HIV-2 } \\
\text { subtype }\end{array}$} & \multirow{2}{*}{$\begin{array}{c}\text { Antiretroviral } \\
\text { regimen }\end{array}$} & \multirow{2}{*}{$\begin{array}{c}\text { Undetectable } \\
\text { VL } \\
\text { (months) }\end{array}$} & \multicolumn{3}{|c|}{ Resistance mutations $^{\mathrm{b}}$} \\
\hline & & & & & & integrase & protease & RT \\
\hline N_1 & M (13) & Equatorial Guinea & B & $A B C+3 T C+R A L$ & no & N155H/E92Q & - & M184V \\
\hline N_2 & M (40) & Guinea Bissau & A & $\mathrm{TDF}+\mathrm{FTC}+\mathrm{DRV} / \mathrm{r}+\mathrm{RAL}$ & no & N155H/E92Q/T97A & I54M/I82F/L90M & M184V \\
\hline F_1 & M (63) & Guinea Bissau & unknown & $\mathrm{TDF}+\mathrm{FTC}+\mathrm{DTG}$ & no & - & - & - \\
\hline F_2 & M (17) & Cabo Verde & $A$ & $\mathrm{ddI}+\mathrm{MVC}+\mathrm{DRV} / \mathrm{r}+\mathrm{RAL}$ & no & N155H/A153G & I54M/I82F/L90M & K65R/Q151M \\
\hline F_3 & $M(51)$ & Mali & A & $\mathrm{TDF}+\mathrm{FTC}+\mathrm{ATV} / \mathrm{r}+\mathrm{RAL}$ & yes (14) & N155H/A153G & I84V/L90M & - \\
\hline F_4 & M (58) & Spain & B & $\mathrm{TDF}+\mathrm{TPV} / \mathrm{r}+\mathrm{RAL}$ & no & N155H/A153G & I54L/I82L & M184V \\
\hline F_5 & M (49) & Senegal & A & $\mathrm{TDF}+\mathrm{FTC}+\mathrm{DRV} / \mathrm{r}+\mathrm{RAL}$ & yes (34) & - & - & M184V \\
\hline F_6 & $F(40)$ & Senegal & A & $\mathrm{TDF}+\mathrm{FTC}+\mathrm{RAL}$ & no & - & - & - \\
\hline F_7 & $M(40)$ & Guinea Bissau & $A$ & $\mathrm{TDF}+\mathrm{DTG}+\mathrm{DRV} / \mathrm{r}$ & no & Q148H/G140S & I50V & K65R \\
\hline F_8 & $F(38)$ & Portugal & $A$ & $\mathrm{TDF}+\mathrm{FTC}+\mathrm{RAL}$ & yes (11) & Q148R/G140A & $\mathrm{I} 82 \mathrm{~L} / \mathrm{I} 84 \mathrm{~V}$ & - \\
\hline F_9 & M (48) & Senegal & A & $\mathrm{DRV} / \mathrm{r}+\mathrm{MVC}+\mathrm{RAL}$ & no & Q148R/G140S & I50V & M184V \\
\hline F_10 & M (53) & Senegal & B & DRV/c + DTG & yes (16) & R263K/E92G & I50V/I54L & K65R \\
\hline F_11 & M (52) & Spain & B & $\mathrm{TDF}+\mathrm{DRV} / \mathrm{r}+\mathrm{RAL}$ & no & Y143C & I54M/I84V & K65R \\
\hline F_12 & $M(47)$ & Africa & A & $\mathrm{ZDV}+3 \mathrm{TC}+\mathrm{RAL}$ & no & Y143C/E92Q & $\mathrm{I} 54 \mathrm{M} / \mathrm{I} 82 \mathrm{~F}$ & K65R/M184V \\
\hline F_13 & M (37) & Guinea Bissau & $A$ & $\mathrm{TDF}+\mathrm{FTC}+\mathrm{RAL}$ & no & Y143G/T97A/A153S & - & K65R/M184V \\
\hline
\end{tabular}

M, male; F, female; VL, viral load; ABC, abacavir; 3TC, lamivudine; ZDV, zidovudine; ddI, didanosine; FTC, emtricitabine; TDF, tenofovir; RAL, raltegravir; DTG, dolutegravir; DRV, darunavir; ATV, atazanavir; LPV, lopinavir; TPV, tipranavir; r, ritonavir; c, cobicistat; MVC, maraviroc.

${ }^{\mathrm{a}} \mathrm{N}$, patient was treatment-naive; F, patient was treatment-experienced.

butations previously found at baseline are shown in bold.

participants were considered to have had a successful response to the predefined primary endpoint, which was a CD4 gain $>100$ cells $/ \mathrm{mm}^{3}$. In the second study, conducted by the University of Washington-Dakar HIV-2 study group, ${ }^{30}$ a total of 30 HIV-2 participants initiated elvitegravir/cobicistat/emtricitabine and tenofovir. Overall, 93.3\% of individuals had viral suppression at week 48 . The median CD4 increase was of +161 cells/ $\mathrm{mm}^{3} .{ }^{30}$ Overall, our results in treatment-naive individuals are in agreement with major findings in these two studies. Most of our patients (16 of $18 ; 88.9 \%$ ) achieved viral suppression with a median CD 4 gain at 1 year of +81 cells $/ \mathrm{mm}^{3}$.

Altogether, treatment outcomes in HIV-2 differ from those seen in HIV-1. For instance, a subset of patients in all three HIV-2 studies (roughly half in our series) had undetectable viraemia at baseline, which almost never occurs in HIV-1. Second, CD4 gains in HIV-2 were relatively modest compared with what is generally seen in treated HIV-1-infected individuals. Lastly, the recognition of frequent selection of drug resistance mutations in HIV-2 individuals that failed virologically, despite low viral load values, must be a matter concern. It supports the overall lower barrier to resistance in HIV-2 compared with HIV-1 for currently available antiretrovirals.

The use of INSTI as part of rescue interventions for HIV-2infected individuals experiencing treatment failure has been examined in prior studies, ${ }^{31-33}$ including some from our group. ${ }^{19,34}$ Benefits are generally seen in both viral suppression and immune recovery, as it was noticed in the present study. However, only half of our 26 HIV-2 pretreated patients that began INSTI achieved and maintained undetectable viraemia for a median of 13 months. While four regained undetectability and rebounded thereafter, the rest failed to achieve undetectable viraemia under INSTI treatment. Moreover, all but three failures selected INSTI resistance-associated mutations.

Regarding resistance patterns at the integrase gene in patients that failed on INSTI, the following mutations were found in 12 patients: N155H (5), Q148H/R (3), Y143C/G (3) and R263K (1). All but one developed other compensatory INSTI mutations including E92Q (4), T97A (2), G140A/S (3) and A153G/S (4). Mutation T97A has been recently reported as a minority resistant variant in one naive HIV-2-infected patient. ${ }^{35}$ By itself, this mutation is not known to confer INSTI resistance, but may reduce susceptibility when present in combination with others.

To our knowledge, this is the first report of R236K plus E92G as a potential mechanism of loss of susceptibility to dolutegravir in HIV-2. The virus was a subtype B variant. In HIV-1 infection, the R263K mutation was originally reported in one individual who failed first-line dolutegravir-based therapy ${ }^{36}$ and another new case has been recently reported. ${ }^{37}$ Further in vitro studies using R263K mutants have shown that it confers a moderate increase in phenotypic resistance to INSTIs along with a drastic reduction in viral replicative capacity. ${ }^{38}$ E92G is a rare non-polymorphic change occasionally selected in patients receiving elvitegravir. ${ }^{17}$ Hypothetically, the combination of R263K and E92G could account for virological failure in our patient.

A recent report has described a new resistance pattern for patients with HIV-2 failing raltegravir. The pattern consists of a five amino acid insertion in the C-terminal region of the integrase gene. ${ }^{39}$ We have not observed this insertion in our patients failing raltegravir. However, this pattern has been identified in another patient failing dolutegravir after prior failure with raltegravir (data not shown). Interestingly, this resistance pattern has been previously described in animal models treated with long-acting 
cabotegravir and resulted in high-level resistance to cabotegravir, dolutegravir, elvitegravir and raltegravir. ${ }^{40}$

Several limitations of our study should be acknowledged, the most relevant being the lack of consideration of drug compliance. This information was reliable for only a subset of patients. For instance, the two native Spaniards who failed treatment were poorly adherent to their medication. However, for most of the rest, who were African immigrants, drug compliance could not be assessed reliably owing to difficulties with language and regular attendance at outpatient appointments. A second limitation of our study is that HIV-1 was not considered in any way for the subset of patients with dual HIV-1 plus HIV-2 infection. However, all of them had undetectable HIV-2 RNA at the end of follow-up. Finally, we used the Sanger sequence instead of next-generation sequencing for the analysis of drug resistance mutations. We identified three failing patients without drug resistance to integrase inhibitors, and minority changes might have been present.

Our study highlights several aspects of HIV-2 ART. First, treatment with INSTIs seems to be safe and effective in HIV-2-infected individuals, particularly in the subset who is drug-naive. In this regard, our findings in real-world patients confirm those obtained in recent controlled studies. Secondly, the proportion of patients with HIV-2 developing resistance mutations to INSTIs after virological failure seems to be high in comparison with the experience with HIV-1, suggesting that the resistance barrier to INSTIs in HIV-2 may be lower. Thirdly, integrase resistance profiles often involved a large number of changes, leading to uncertainty about crossresistance, even for the most recent INSTIs such as bictegravir and cabotegravir.

Given that the clinical management of HIV-2-infected patients should follow the rules of HIV-1 infection, early diagnosis and treatment initiation is recommended. However, owing to the limited therapeutic armamentarium for HIV-2 compared with HIV-1, this population must be closely followed-up, bearing in mind that rescue interventions may be challenging.

\section{Acknowledgements}

The authors thank all members of the Spanish HIV-2 Network for their participation.

\section{Members of the HIV-2 Spanish Study Group}

C. Rodríguez, M. Vera and J. del Romero (Centro Sanitario Sandoval, Madrid); G. Marcaida and M. D. Ocete (Hospital General Universitario, Valencia); E. Caballero (Hospital Vall d'Hebrón, Barcelona); A. Aguilera (Hospital ConxoCHUS, Santiago); R. Benito (Hospital Clínico Universitario Lozano Blesa, Zaragoza); R. Ortiz de Lejarazu and S. Rojo (Hospital Clínico Universitario, Valladolid); J. M. Eirós and C. Ramos (Hospital Rio Hortega, Valladolid); J. García and I. Paz (Hospital Cristal-Piñor, Orense); M. Trigo, J. Diz and M. García-Campello (Complejo Hospitalario, Pontevedra); M. Rodríguez-Iglesias (Hospital Universitario, Puerto Real); A. Hernández-Betancor and A. M. Martín (Hospital Insular Hospital Universitario, Las Palmas de Gran Canaria); J. M. Ramos and A. Gimeno (Hospital Universitario, Alicante); V. Sánchez (Hospital General, Elche); C. Gómez-Hernando (Complejo Hospitalario Virgen de la Salud, Toledo); G. Cilla and E. Pérez-Trallero (Hospital Donostia, San Sebastián); L. Fernández-Pereira (Hospital San Pedro de Alcántara, Cáceres); J. Niubó (Ciudad Sanitaria de Bellvitge, Barcelona); M. Hernández, A. M. López-Lirola and J. L. Gómez-Sirvent (Hospital Universitario La Laguna, Tenerife); L. Force (Hospital General, Mataró); J. Cabrera, S. Pérez and L. Morano (Hospital do Meixoeiro, Vigo); C. Raya (Hospital del Bierzo,
Ponferrada); A. González-Praetorius (Hospital Universitario, Guadalajara); C. Cifuentes and M. Peñaranda (Hospital Son Espases, Mallorca); M. C. Nieto (Hospital de Basurto, Bilbao); J. M. Montejo (Hospital de Cruces, Bilbao); L. Roc (Hospital Miguel Servet, Zaragoza); I. Viciana (Hospital Virgen de la Victoria, Málaga); A. B. Lozano, E. Fernández-Fuertes and J. M. Fernández (Hospital de Poniente, Almería); I. García-Bermejo and G. Gaspar (Hospital Universitario, Getafe); R. Téllez and M. Górgolas (Fundación Jiménez Díaz, Madrid); P. Miralles, L. Pérez, M. Valeiro and T. Aldamiz (Hospital Gregorio Marañón, Madrid); N. Margall (Hospital Santa Creu i Sant Pau, Barcelona); A. Suárez and I. Rodríguez-Avial (Hospital Clínico San Carlos, Madrid); S. Requena, L. Benítez-Gutiérrez, V. Cuervas-Mons and C. de Mendoza (IIS Hospital Universitario Puerta de Hierro, Majadahonda); P. Barreiro (Hospital Universitario La Paz, Madrid); and V. Soriano (UNIR Health Sciences School and Hospital Universitario La Paz, Madrid).

\section{Funding}

This work was supported in part by grants from: Fundación Investigación y Educación en SIDA; FIPSE (36742/08); Fondo de Investigación Sanitaria-Fondos- FEDER (FIS EC10/277, CES12/003, FI14/00264, CD14/ 00243) and European Collaborative HIV and Anti-HIV Drug Resistance Network (CHAIN, project FP7-223131).

\section{Transparency declarations}

None to declare.

\section{References}

1 Clavel F, Guetard D, Brun VF et al. Isolation of a new human retrovirus from West African patients with AIDS. Science 1986; 233: 343-6.

2 Centers for Disease Control and Prevention. HIV-2 infection surveillanceUnited States, 1987-2009. MMWR Morb Mortal Wkly Rep 2011; 60: 985-8.

3 Sousa J, Temudo M, Hewlett B et al. Male circumcision and the epidemic emergence of HIV-2 in West Africa. PLoS One 2016; 11: e0166805.

4 Soriano V, Tor J, Ribera A et al. HIV-1, HIV-2 and HTLV-I infection in highrisk groups in Spain. AIDS 1989; 3: 615-6.

5 De Mendoza C, Cabezas T, Caballero E et al. HIV type 2 epidemics in Spain: challenges and missing opportunities. AIDS 2017; 31: 1353-64.

6 Gilleece Y, Chadwick D, Breuer J et al. British HIV Association guidelines for antiretroviral treatment of HIV-2 positive individuals 2010. HIV Med 2010; 11: 611-9.

7 Menéndez-Arias L, Alvarez M. Antiretroviral therapy and drug resistance in human immunodeficiency virus type 2 infection. Antiviral Res 2014; 102: 70-86.

8 Poveda E, Rodés B, Toro C et al. Are fusion inhibitors active against all HIV variants? AIDS Res Hum Retroviruses 2004; 20: 347-8.

9 Raugi D, Smith R, Ba S et al. Complex patterns of protease inhibitor resistance among antiretroviral treatment experienced HIV-2 patients from Senegal: implications for second-line. Antimicrob Agents Chemother 2013; 57: 2751-60.

10 Desbois D, Roquebert B, Peytavin G et al. In vitro phenotypic susceptibility of human immunodeficiency virus type 2 clinical isolates to protease inhibitors. Antimicrob Agents Chemother 2008; 52: 1545-8.

11 Ekouevi D, Tchounga B, Coffie $\mathrm{P}$ et al. Antiretroviral therapy response among HIV-2 infected patients: a systematic review. BMC Infect Dis 2014; 14: 461.

12 Azijn H, Tirry I, Vingerhoets J et al. TMC278, a next-generation non-nucleoside reverse transcriptase inhibitor (NNRTI), active against wild-type and NNRTI-resistant HIV-1. Antimicrob Agents Chemother 2010; 54: 718-27. 
13 Treviño A, de Mendoza C, Caballero E et al. Drug resistance mutations in patients infected with HIV-2 living in Spain. J Antimicrob Chemother 2011; 66: 1484-8.

14 Cavaco-Silva J, Abecasis A, Miranda A et al. HIV-2 integrase polymorphisms and longitudinal genotypic analysis of HIV-2 infected patients failing a raltegravir-containing regimen. PLoS One 2014; 9: e92747.

15 Andreatta K, Miller M, White K. HIV-2 antiviral potency and selection of drug resistance mutations by the integrase strand transfer inhibitor elvitegravir and NRTIs emtricitabine and tenofovir in vitro. J Acquir Immune Defic Syndr 2013; 62: 367-74.

16 Charpentier C, Roquebert B, Delelis O et al. Hot spots of integrase genotypic changes leading to HIV-2 resistance to raltegravir. Antimicrob Agents Chemother 2011; 55: 1293-5.

17 Treviño A, Caballero E, de Mendoza C et al. The burden of neglected HIV2 and HTLV-1 infections in Spain. AIDS Rev 2015; 17: 212-9.

18 Ferns R, Garson J. Development and evaluation of a real-time RT-PCR assay for quantification of cell-free HIV type 2 using a brome mosaic virus internal control. J Virol Methods 2006; 135: 102-8.

19 Treviño A, Cabezas T, Lozano A et al. Dolutegravir for the treatment of HIV-2 infection. J Clin Virol 2015; 64: 12-5.

20 Shafer R. Rationale and uses of a public HIV drug resistance database. J Infect Dis 2006; 194: 551-8.

21 Wensing A, Calvez V, Gunthard H et al. 2017 update of the drugresistance mutations in HIV-1. Topics Antivir Ther 2017; 24: 132-3.

22 Charpentier C, Camacho R, Ruelle J et al. HIV-2 EU supporting standardized HIV-2 drug resistance interpretation in Europe: an update. Clin Infect Dis 2015; 61: 1346-7.

23 Saag M, Benson C, Gandhi R et al. Antiretroviral drugs for treatment and prevention of HIV infection in adults: 2018 recommendations of the international AIDS antiviral society USA panel. JAMA 2018; 320: 379-96.

24 US Department of Health and Human Services. Guidelines for the Use of Antiretroviral Agents in HIV-1 Infected Adults and Adolescents. 2018. https:// aidsinfo.nih.gov/guidelines.

25 Gottlieb G. Treatment of HIV-2 Infection. 2018. www.uptodate.com/con tents/treatment-of-hiv-2-infection.

26 Roquebert B, Damond F, Collin G et al. HIV-2 integrase gene polymorphism and phenotypic susceptibility of HIV-2 clinical isolates to the integrase inhibitors raltegravir and elvitegravir in vitro. J Antimicrob Chemother 2008; 62: 914-20.

27 Smith R, Raugi D, Pan C et al. In vitro activity of dolutegravir against wildtype and integrase inhibitor-resistant HIV-2. Retrovirology 2015; 12: 10.
28 Tsiang M, Jones G, Goldsmith J et al. Antiviral activity of bictegravir (GS9883), a novel potent HIV-1 integrase strand transfer inhibitor with an improved resistance profile. Antimicrob Agents Chemother 2016; 60: 7086-97.

29 Matheron S, Descamps D, Gallien S et al. First-line raltegravir/emtricitabine/tenofovir combination in HIV-2 infection: a phase 2, non-comparative trial (ANRS 159 HIV-2). Clin Infect Dis 2018; 67: 1161-7.

30 Ba S, Raugi D, Smith R et al. A trial of a single-tablet regimen of elvitegravir, cobicistat, emtricitabine, and tenofovir disoproxil fumarate for the initial treatment of HIVinfection in a resource-limited setting: 48 week results from Senegal, West Africa. Clin Infect Dis 2018; 67: 1588-94.

31 Charpentier C, Larrouy L, Collin G et al. In-vitro phenotypic susceptibility of HIV-2 clinical isolates to the integrase inhibitor S/GSK1349572. AIDS 2010; 24: $2753-5$.

32 Smith R, Raugi D, Pan C et al. Three main mutational pathways in HIV-2 lead to high-level raltegravir and elvitegravir resistance: implications for emerging HIV-2 treatment regimens. PLoS One 2012; 7: e45372.

33 Descamps D, Peytavin G, Visseaux B et al. Dolutegravir in HIV-2 infected patients with resistance virus to first-line integrase inhibitors from the French named patient program. Clin Infect Dis 2015; 60: 1524-7.

34 Requena S, Treviño A, Cabezas T et al. Drug resistance mutations in HIV-2 failing raltegravir and influence on dolutegravir response. J Antimicrob Chemother 2017; 72: 2083-8.

35 Storto $A$, Visseaux B, Bertine $M$ et al. Minority resistant variants are also present in HIV-2-infected antiretroviral-naive patients. J Antimicrob Chemother 2018; 73: 1173-6.

36 Quashie P, Mesplède T, Han Y et al. Characterization of the R263K mutation in HIV-1 integrase that confers low-level resistance to the secondgeneration integrase strand transfer inhibitor dolutegravir. J Virol 2012; 86: 2696-705.

37 Pena M, Chueca N, Hernández-Febles M et al. Use of next generation sequencing to study a first line failure to a dolutegravir containing regimen. 16th European Meeting on HIV \& Hepatitis: Treatment Strategies \& Antiviral Drug Resistance, 2018. Abstract 31. Rome, Italy.

38 Mesplede T, Quaashie P, Osman N et al. Viral fitness cost prevents HIV-1 from evading dolutegravir drug pressure. Retrovirology 2013; 10: 22.

39 Le Hingrat Q, Collin G, Lê M et al. A new mechanism of resistance of HIV-2 to integrase inhibitors: a 5 amino-acids insertion in the integrase C-terminal domain. Clin Infect Dis 2018; doi:10.1093/cid/ciy940.

40 Andrews CD, Mohri H, Bernard L et al. Evaluation of resistance to cabotegravir long-acting (CAB LA) in SIVmac251-infected macaques. AIDS Res Hum Retroviruses 2016; 32: 43. 